\title{
Is Schizophrenia a Neurodegenerative Disease? Evidence from Age-Related Decline of Brain-Derived Neurotrophic Factor in the Brains of Schizophrenia Patients and Matched Nonpsychiatric Controls
}

\author{
Jagadeesh Rao ${ }^{a}$ Joshua Chiappelli ${ }^{b}$ Peter Kochunov ${ }^{b}$ William T. Regenold ${ }^{c}$ \\ Stanley I. Rapoport ${ }^{\mathrm{a}} \quad$ L. Elliot Hong ${ }^{\mathrm{b}}$ \\ a Brain Physiology and Metabolism Section, Laboratory of Neurosciences, National Institute on Aging, National Institutes \\ of Health, Bethesda, Md., and ${ }^{b}$ Maryland Psychiatric Research Center and ' Division of Geriatric Psychiatry, Department of \\ Psychiatry, University of Maryland School of Medicine, Baltimore, Md., USA
}

\section{Key Words}

Aging · Brain · Prefrontal cortex - White matter ·

Schizophrenia $\cdot$ Postmortem $\cdot$ Brain-derived

neurotrophic factor

\begin{abstract}
Background: Brain-derived neurotrophic factor (BDNF) protein levels decline in the brain during senescence and are also shown to be reduced in schizophrenia patients. BDNF is present in both the gray and white matters of the brain. It is unclear whether BDNF abnormalities in schizophrenia are specific to gray and/or white matter. Objective: We hypothesized that the age-related BDNF decline is abnormal and contributes to the reduced BDNF in schizophrenia. Methods: We tested this hypothesis by measuring BDNF protein levels in postmortem gray and white matter, using the prefrontal cortex (PFC) and the genu of the corpus callosum as regions of interests, from 20 schizophrenia patients and 20 matched nonpsychiatric controls. Samples were selected across the adult lifespan - from 20 to 80 years of age. Results: PFC gray matter BDNF protein levels were significantly lower in older age in both nonpsychiatric comparisons and patients, while BDNF in white matter did not decrease significantly with age
\end{abstract}

(c) 2014 S. Karger AG, Base

$1660-2854 / 14 / 0151-0038 \$ 39.50 / 0$ in either group. PFC BDNF was linearly lower from 20 to 80 years of age in nonpsychiatric comparisons. In schizophrenia, the age effect was similarly linear in younger patients but a decline did not occur in older patients. Conclusion: PFC BDNF does not follow a normative linear age effect in schizophrenia patients as they grow older, which may represent a 'floor effect' due to earlier decline or a survivor cohort of older patient donors who are less susceptible to a schizophrenia-related pathological aging process.

(c) 2014 S. Karger AG, Basel

\section{Introduction}

Whether schizophrenia follows a neurodegenerative course has been debated since Kraepelin [1], who referred to a progressive decline in neurobiological and clinical characteristics as patients grow older. Evidence remains inconsistent $[2,3]$, ranging from confirming a progressive decline [4] to identifying an initial decline followed by stability [3] or even improvement [5] as patients age. A hallmark of aging is a decline in neuronal plasticity, with a change in brain-derived neurotrophic factor (BDNF) being one of the potential mechanisms [6], al-

\section{KARGER 125}

E-Mail karger@karger.com

www.karger.com/ndd
Dr. L. Elliot Hong

Maryland Psychiatric Research Center

55 Wade Avenue

Baltimore, MD (USA)

E-Mail ehong@mprc.umaryland.edu 
though BDNF is also known to be associated with many other pathophysiological processes. Our aim here was to test whether BDNF may serve as a marker to delineate the neurodegeneration across the adult lifespan of schizophrenia patients by testing the hypothesis that the agerelated BDNF decline is abnormal and contributes to the reduced BDNF in schizophrenia.

BDNF is a neurotrophin widely distributed in the adult human brain. Its effects are largely mediated by the high-affinity receptor $\operatorname{TrkB}$ (tropomyosin-related kinase) that promotes memory, cell survival, synaptic excitation, and neuronal plasticity $[7,8]$. BDNF and TrkB play important roles in brain aging $[6,9,10]$ and reduced levels of BDNF, as found in postmortem brains, are thought to contribute to age-related memory loss in early Alzheimer's disease and mild cognitive impairment [11]. In animal aging studies, old age is associated with reduced $\mathrm{BDNF}$ expression in the prefrontal cortex (PFC) and hippocampus [6]. Therefore, BDNF may be a useful biomarker to reexamine the putative neurodegenerative course in schizophrenia.

Prior studies indicate that BDNF is abnormally reduced in schizophrenia. We reported reduced BDNF mRNA and protein levels in the PFC of 10 schizophrenia patients compared with 10 age-matched nonpsychiatric comparisons (no overlap with the current sample) [12], consistent with other postmortem studies $[13,14]$. These findings are further supported by clinical evidence showing reduced serum BDNF in many but not all studies in schizophrenia, including reduced serum BDNF in treatment-naïve and first-episode schizophrenia patients [15-17]. However, none of the previous studies measured BDNF systematically across the full adult lifespan of schizophrenia patients, which was the primary aim of this study. Furthermore, we designed this study to compare BDNF over the adult lifespan in PFC gray matter with that in white matter because age-related variations in BDNF may be different in gray versus white matter in schizophrenia, although this has not been reported previously. We examined postmortem brains from schizophrenia patients compared with nonpsychiatric comparisons using an age-cohort design to include schizophrenia patient donors from 20 to 80 years of age. Tissues were selected using five 12-year age bins, matching patients and nonpsychiatric comparisons on gender, postmortem interval (PMI), $\mathrm{pH}$, and RNA integrity number (RIN) as closely as possible within each age bin. As a result, we had well-matched casecontrol samples evenly weighted across 60 years of the adult lifespan.

BDNF in the Brains of Schizophrenia Patients and Nonpsychiatric Controls

\section{Materials and Methods}

\section{Brain Tissue}

Postmortem brains $(n=40)$, stored at $-80^{\circ} \mathrm{C}$, were obtained from the Maryland Brain Collection (http://www.mprc.umary land.edu/mbc.asp). The brains were slowly warmed to $-20^{\circ} \mathrm{C}$, and gray matter from the PFC and white matter at the genu of the corpus callosum were dissected. The genu was selected because it contains the primary interhemispheric white matter fiber bundle connecting the left and right PFC. The PFC was identified at the junction of Brodmann areas 9 and 10, adjacent to area 46, which is the center of the dorsolateral PFC. The genu was dissected from the dorsal curvature portion of the anterior of the corpus callosum, inferior to the anterior cingulate cortex. Visible gray matter and vascular tissues were carefully avoided or removed. Dissections were alternated between the left and right hemispheres. The specimens included 20 schizophrenia donors (10 had taken first-generation antipsychotic medications, 4 had taken second-generation antipsychotics, 5 had taken two or more antipsychotic medications, and 1 had not taken antipsychotics for 6 months or more before death). Nonpsychiatric comparison specimens were from individuals with no history of psychosis, mood disorders, drug dependence, psychiatric treatment, or hospitalization, as determined by medical examiner documentation and telephone screening of the next of kin. Specimens were selected as case-control pairs within each 'age bin' of 12 years and were frequency matched on sex, age, PMI, pH, and RIN. For example, these variables were frequency matched between patients and nonpsychiatric comparisons as closely as possible within the age bin of 20-31 years of age. We then moved the matching procedures to the next age bin of $32-43$, and then $44-55,56-67$, and finally $68-80$ years of age. Clinical information was obtained through family interviews, including the Structured Clinical Interview for DSM-IV Axis I Disorders (SCIDIV). Two senior psychiatrists performed best-estimate diagnoses using the Diagnostic Evaluation after Death [18] in conjunction with a review of past medical and psychiatric records. Postmortem clinical diagnoses were established according to the method developed by Roberts et al. [19]. All specimens were obtained with informed consent of the legal next of kin with approval from the University of Maryland IRB, and the study was approved by the Office of Human Subject Research, National Institutes of Health.

The tight matching, using age cohorts across a large lifespan, resulted in excellent frequency-matched samples on key tissue characteristics (table 1). For quality assessment, tissue quality depends on high tissue $\mathrm{pH}$ and RIN and no agonal factors [20-22]. In the 40 cases and nonpsychiatric comparisons selected for this study, causes of death included cardiovascular causes $(n=16)$, motor vehicle accident $(n=1)$ and accidental drowning $(n=3)$ in the nonpsychiatric comparison donors, and suicide by drowning, jumping or overdose $(n=7)$, cardiovascular causes $(n=11)$, and other causes $(n=1)$ in the patient donors. We matched PMI, $\mathrm{pH}$ and RIN between patients and nonpsychiatric comparisons (table 1). $\mathrm{pH}$ was measured before dissection using cerebellar tissue from frozen brain; $\mathrm{pH}$ from each dissected tissue was not separately assayed. The tissue was homogenized for $1 \mathrm{~min}$ so as not to raise the temperature of the sample, and the $\mathrm{pH}$ probe from the Omni PCR tissue homogenizing kit (OMNI International, Kennesaw, Ga., USA) was then inserted to measure the pH. RIN was obtained from the entire Agilent electrophoretic trace with a RIN software algorithm on a scale of $1-10$, with 1 
Table 1. Demographics and tissue quality

\begin{tabular}{|c|c|c|c|c|c|c|c|}
\hline $\begin{array}{l}\text { Age cohort, } \\
\text { years }\end{array}$ & Diagnosis & $\mathrm{n}$ & Age, years & Sex (F:M) & PMI, h & $\mathrm{pH}$ & RIN \\
\hline $20-31$ & nonpsychiatric comparison & 4 & $22.8 \pm 3.6$ & $0: 4$ & $18.2 \pm 6.1$ & $6.7 \pm 0.2$ & $8.3 \pm 1.1$ \\
\hline \multirow[t]{2}{*}{$32-43$} & nonpsychiatric comparison & 4 & $36.0 \pm 5.3$ & $1: 3$ & $10.0 \pm 5.2$ & $6.7 \pm 0.3$ & $9.4 \pm 0.3$ \\
\hline & schizophrenia & 4 & $38.0 \pm 4.8$ & $0: 4$ & $17.5 \pm 7.6$ & $6.7 \pm 0.3$ & $9.1 \pm 0.7$ \\
\hline $44-55$ & schizophrenia & 4 & $48.2 \pm 6.2$ & $1: 3$ & $11.5 \pm 4.0$ & $6.9 \pm 0.3$ & $8.1 \pm 0.5$ \\
\hline \multirow[t]{2}{*}{$56-67$} & nonpsychiatric comparison & 4 & $61.2 \pm 5.4$ & $0: 4$ & $12.2 \pm 6.1$ & $6.9 \pm 0.3$ & $8.9 \pm 0.8$ \\
\hline & schizophrenia & 4 & $58.5 \pm 2.4$ & $0: 4$ & $14.2 \pm 8.6$ & $7.0 \pm 0.5$ & $8.5 \pm 0.8$ \\
\hline \multirow[t]{2}{*}{$68-80$} & nonpsychiatric comparison & 4 & $73.2 \pm 7.1$ & $0: 4$ & $10.5 \pm 5.4$ & $6.7 \pm 0.1$ & $8.9 \pm 0.2$ \\
\hline & schizophrenia & 4 & $72.0 \pm 3.5$ & $0: 4$ & $9.5 \pm 7.0$ & $6.8 \pm 0.2$ & $8.8 \pm 0.1$ \\
\hline Combined & $\mathrm{p}$ value & & 0.93 & 1.00 & 0.55 & 0.36 & 0.35 \\
\hline
\end{tabular}

PMI, pH and RIN assess different aspects of tissue quality.

being the lowest and 10 the highest RNA quality (Agilent Bioanalyzer 2100; Agilent Technologies, Santa Clara, Calif., USA) [23].

\section{BDNF Assay}

Cytosolic and membrane extracts were prepared from gray and white brain tissue separately, using methods previously described [12]. Briefly, tissue was homogenized in a buffer containing $20 \mathrm{mM}$ Tris- $\mathrm{HCl}$ (pH 7.4), 2 mM EGTA, 5 mM EDTA, $1.5 \mathrm{~mm}$ pepstatin, $2 \mathrm{~mm}$ leupeptin, $0.5 \mathrm{~mm}$ phenylmethylsulfonyl fluoride, $0.2 \mathrm{U} / \mathrm{ml}$ aprotinin and $2 \mathrm{mM}$ dithiothreitol. The homogenate was centrifuged at $100,000 \mathrm{~g}$ for $60 \mathrm{~min}$ at $4^{\circ} \mathrm{C}$. The resulting supernatant was the cytosolic fraction, and the pellet was resuspended in the homogenizing buffer containing $0.2 \%(\mathrm{w} / \mathrm{v})$ Triton $\mathrm{X}-100$. The suspension was kept at $4^{\circ} \mathrm{C}$ for 60 min with occasional stirring and then centrifuged at $100,000 \mathrm{~g}$ for $60 \mathrm{~min}$ at $4^{\circ} \mathrm{C}$. The resulting supernatant was the membrane fraction. Overall protein concentrations of the membrane and cytosolic fractions were determined with Bio-Rad protein reagent (Bio-Rad, Hercules, Calif., USA). BDNF protein levels were measured from brain cytosolic extracts $(75 \mu \mathrm{g})$ using an ELISA kit as suggested by the manufacturer (Neo Bio Labs, Cambridge, Mass., USA). This kit determines all the isoforms of BDNF and differentiates the BDNF isoforms. Values are expressed in picograms per milligram. More details have been described previously [12]. All assays were performed blind to diagnosis and demographic information.

\section{Statistical Analysis}

The matching of age here does not equate to a paired sample design because the aim is not to 'remove' the effect of age so that the outcome of interest between the two groups would be examined without this variable. Here, we hypothesized that the effect of age on $\mathrm{BDNF}$ or the underlying mechanism driving age-related changes in BDNF is different in schizophrenia patients and normal controls. Therefore, we drew samples from two independent groups of brains, structured using age bins so that each sample was distributed approximately uniformly across the full range of ages. This is a classic way to test the 'constant difference across ages' assumption: to identify the age-related trends and their differences between groups. We used an ANOVA model where tissue type (gray vs. white matter) was the repeated measure and age bin (five age cohorts) and diagnosis (schizophrenia vs. control) were the between-subject factors.

This model is based on linear distribution. Initial observation of the data suggested that the age distribution in the gray matter may not be linear. To test whether the models for linear versus quadratic age trends in BDNF in schizophrenia patients and controls were significantly different, we fitted another regression equation that included diagnosis, age, age ${ }^{2}$, and their interaction terms. Diagnosis was coded as -1 and 1 in the model so that we could directly compare the age-related coefficients between the groups. The model would also test whether there were group differences between linear and quadratic age terms. Significant tests of main effect or interaction were followed by post hoc tests.

\section{Results}

The ANOVA showed no significant effect of diagnosis $\left(\mathrm{F}_{1,30}=1.14, \mathrm{p}=0.29\right)$ but a significant main effect of age $\left(\mathrm{F}_{4,30}=5.29, \mathrm{p}=0.002\right)$, a significant age $\times$ loca-
40
Neurodegener Dis 2015;15:38-44 DOI: $10.1159 / 000369214$
Rao/Chiappelli/Kochunov/Regenold/ Rapoport/Hong 
Fig. 1. Aging trends of $\mathrm{BDNF}$ in the $\mathrm{PFC}$ in nonpsychiatric controls $(n=20)$ and schizophrenia patients $(n=20)$ from age 20 to 80 years.

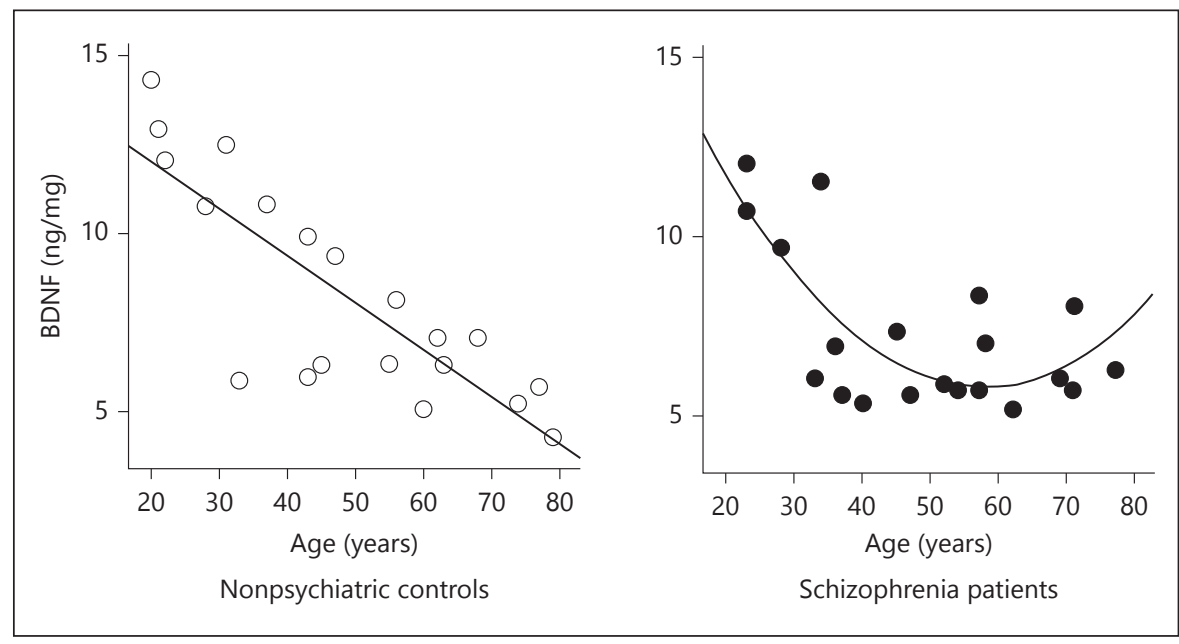

tion interaction $\left(\mathrm{F}_{4,30}=4.05, \mathrm{p}=0.010\right)$ and an insignificant location $\times$ diagnosis interaction $\left(\mathrm{F}_{1,30}=3.14\right.$, $\mathrm{p}=0.086)$. There was no significant location $\times$ diagnosis $\times$ age interaction $(p=0.72)$. The significant age $\times$ location interaction was examined by Pearson's correlation in each location. BDNF levels had a significant age effect in gray matter $(\mathrm{r}=-0.82, \mathrm{p}<0.001$ and $\mathrm{r}=-0.58$, $\mathrm{p}=0.01$ in controls and patients, respectively), which remained significant after Bonferroni correction for four tests $(\mathrm{p}<0.012)$, but not in white matter $(\mathrm{r}=0.05$, $\mathrm{p}=0.82$ and $\mathrm{r}=0.13, \mathrm{p}=0.57$ in controls and patients, respectively; fig. 1). Therefore, the primary findings were the following: (1) a robust, gray matter-specific, linear age effect of BDNF protein level in normal nonpsychiatric comparisons from age 20 all the way to 80 years and (2) a similar but less robust linear age effect in schizophrenia patients.

Further exploration of the data suggested that the relationship between the dorsolateral PFC BDNF and age might have two trends depending on younger versus older age rather than quadratic in schizophrenia patients (fig. 2). We explored this post hoc hypothesis by dividing the groups into two 30-year cohorts: $20-50$ and $50-80$ years of age. In the younger age cohort of 20-50 years, PFC BDNF levels became lower with age similarly in nonpsychiatric comparisons and patients (fig. 2). A comparison of their slopes [24] revealed no statistical difference $(p=0.93)$. In this age range, patients showed a trend of reduced PFC BDNF compared with nonpsychiatric comparisons $(\mathrm{F}=2.81$, d.f. $=19, \mathrm{p}=0.12)$. In the older age cohort of 50-80 years, PFC BDNF levels declined in nonpsychiatric comparisons but not in patients, and the slopes were significantly different $(p=0.038)$. There was

BDNF in the Brains of Schizophrenia Patients and Nonpsychiatric Controls

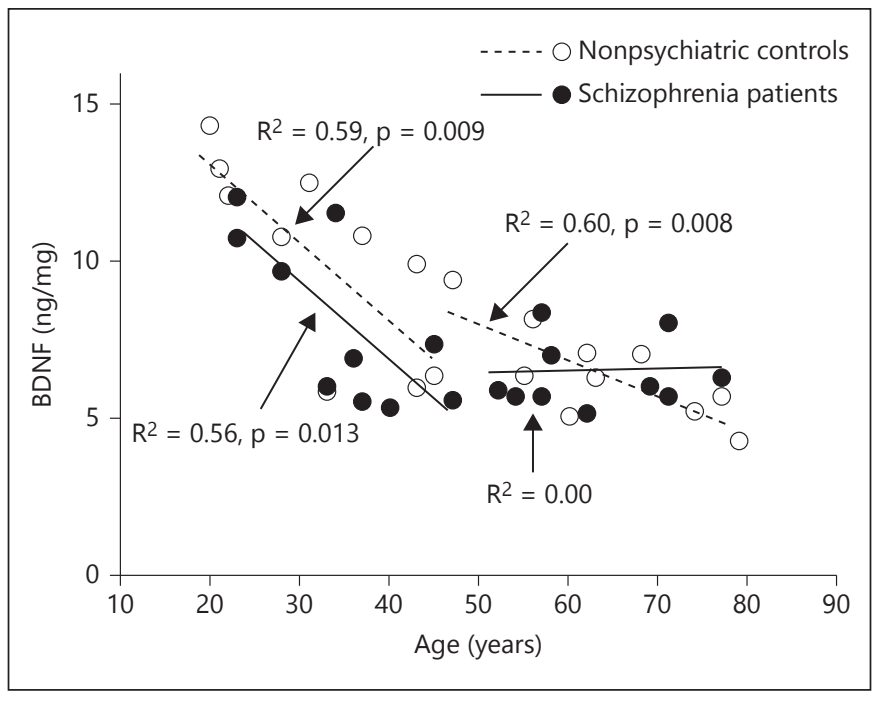

Fig. 2. Exploratory, secondary analysis using a bimodal interpretation of figure 1 when splitting the groups into two 30 -year cohorts (20-50 and 50-80 years of age). Here, the slopes of decline between the patients and nonpsychiatric comparisons were similar in younger age but statistically different in older age.

no significant group difference in PFC BDNF in this age range $(\mathrm{p}=0.90)$.

For white matter BDNF, there was no significant correlation between the BDNF level and age in nonpsychiatric comparisons $(r=0.05, p=0.82)$ or schizophrenia patients $(r=0.13, p=0.57)$ and thus no evidence of a significant age-related decline in BDNF in either group in the genu of corpus callosum.

Compared with all nonpsychiatric controls, BDNF protein levels were lower in the dorsolateral PFC of 


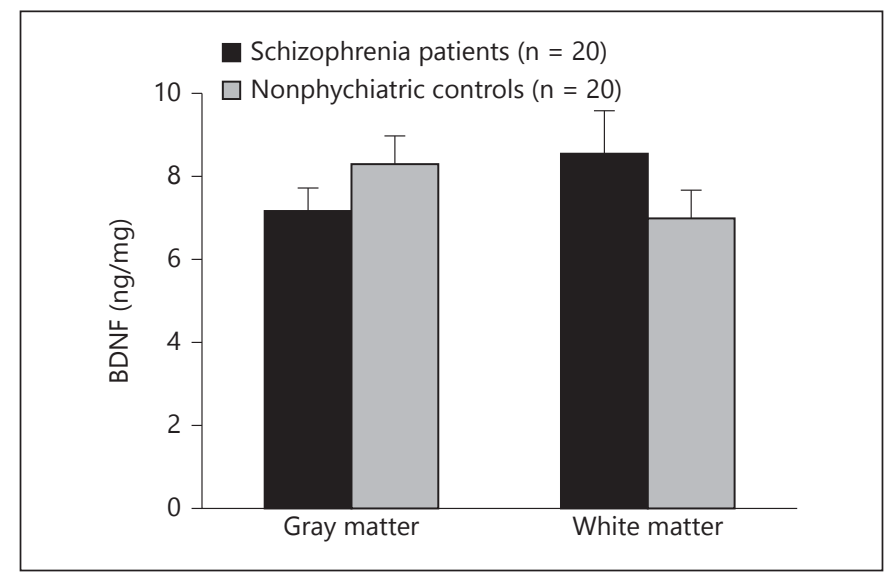

Fig. 3. BDNF protein levels (mean and SEM) in PFC gray matter and the genu of corpus callosum white matter from nonpsychiatric comparisons and schizophrenia patients. BDNF protein level was numerically lower in the PFC of schizophrenia patients $(p=0.20)$ but higher in the genu of schizophrenia patients $(p=0.19)$ compared with nonpsychiatric controls, although none was statistically significant.

schizophrenia patients $(\mathrm{p}=0.20)$. In contrast, BDNF protein levels were higher in the genu of schizophrenia patients $(\mathrm{p}=0.19)$. This may explain the trend of a location $\times$ diagnosis interaction $(\mathrm{p}=0.086)$, although none was statistically significant (fig. 3 ).

Concerning potential confounds by differences of PMI, $\mathrm{pH}$ or RIN in BDNF levels, we found that PMI was significantly correlated with PFC BDNF level $(r=0.47$, $\mathrm{p}=0.04)$ in the nonpsychiatric controls. Note that this was in the positive direction and was not significant with correction of six correlation analyses. Other than this, we found no other significant correlation between PMI, $\mathrm{pH}$ or RIN and BDNF at the PFC or genu in either nonpsychiatric controls or patients (all $r<0.39$, all $p>0.09$ ). Incidentally, older age was associated with shorter PMI $(\mathrm{r}=$ $-0.44, p=0.004)$ although older age was not significantly associated with $\mathrm{pH}(\mathrm{r}=0.23, \mathrm{p}=0.15)$ or $\mathrm{RIN}(\mathrm{r}=-0.10$, $\mathrm{p}=0.59)$ in this sample.

\section{Discussion}

This study examined BDNF levels across a large span of adult life from 20 to 80 years of age in the postmortem PFC and white matter genu, and tested whether BDNF may serve as a biomarker for the putative neurodegenerative course of schizophrenia. We found a statistically significant age effect of BDNF in both nonpsychiatric controls and schizophrenia patients in the PFC but not in the genu. These aging trends appeared convincing because they were observed in nonpsychiatric comparisons and in patients - two independent samples. One difference was that the age effect of BDNF in the PFC followed a relatively continuous, linear decline from age 20 to 80 in the nonpsychiatric controls, while in schizophrenia patients we observed a bimodal relationship - a similar linear decline in younger age, but no further decline in the older-age patients.

Several mechanisms may explain the lack of further reduction of PFC BDNF in older-age schizophrenia patients. Firstly, because patients showed a trend of reduced PFC BDNF compared with nonpsychiatric comparisons in younger age while the slope was similar to that of the nonpsychiatric comparisons, BDNF levels of the patients could have reached a 'floor effect' sooner, which could explain the lack of further decline in the older-age patients.

Secondly, medication may have played a role. We previously reported that the antipsychotic medication clozapine increased BDNF in the rat brain [25]. This observation is replicated in both animals and humans using different antipsychotic medications [26-28]. Further, the current sample consisted of mostly male patients and the BDNF elevating effect of antipsychotic medication could be more robust in male patients [29]. The older the age, the more likely the patients were to have received antipsychotics for a longer time period and thus the more dampened the decline of BDNF. However, because the normal course of BDNF could be an age-related decline, it may be difficult to imagine that antipsychotic medication could not only reverse the reduced $\mathrm{BDNF}$ in patients but also halt the normative BDNF decline. Definitive testing of this hypothesis may require comparing postmortem tissue samples of older schizophrenia patient donors who are treatment naïve with nondonors who are on antipsychotic medication; tissue from either would be difficult to acquire.

Thirdly, aging in schizophrenia itself could lessen the impact of the disease, such that BDNF decline may slow down as patients grow older. A similar proposition has been suggested based on some limited clinical evidence [5]. However, because normal aging may be associated with a linear BDNF decline, a lack of decline does not seem consistent with the 'normalization' argument.

Fourthly, a survivor effect may be the most likely explanation. Because the lifespan of schizophrenia patients is about 20 years shorter than the general population [30-33], patients who survive into older age to donate their brains are more likely to be individuals who possess
Rao/Chiappelli/Kochunov/Regenold/ Rapoport/Hong 
protective mechanisms such as a reduced age-related decline in BDNF, which is not shared by earlier-dying patients. Such a hypothetical survivor effect would be more prominent as a donor's age increases. Interestingly, this interpretation implies that there may be an abnormal brain aging process in schizophrenia patients and that the BDNF level could be a 'protective biomarker' for the minority of schizophrenia patients who survive into old age.

Support for the survivor effect explanation can be found in contrasting results from BDNF studies in patients with major depressive disorder (MDD). In a postmortem study of a similar sample size of $21 \mathrm{MDD}$ patients and 21 controls across an age range of $16-74$ years, BDNF gene expression showed consistent age-related declines. However, MDD patients had a steeper decline compared with controls [34], which is in contrast to our finding in schizophrenia. Note that after accounting for suicide, older MDD patients have normal life expectancy [35]. Therefore, in a psychiatric illness with normal life expectancy (excluding suicide), the brain BDNF level followed the same or even a steeper trend of normative age-related decline. However, in schizophrenia, with a shorter life expectancy even after excluding suicide, the BDNF level did not show a normative decline. This comparison with MDD further suggests that the blunted or halted normal decline of BDNF in the PFC of older schizophrenia patients may not be a disease effect but rather a bias from donor cohorts who are 'survived' patients and/or a possible alteration by history of antipsychotic medication treatment.

BDNF in the genu white matter did not have a significant age effect in either nonpsychiatric comparisons or patients, in contrast with the strong age effects in the gray matter. It is unclear whether this is similar in other white matter areas of the brain. One study found a negative relation between plasma BDNF and white matter integrity on MRI in older adults, and suggested that plasma BDNF levels reflect repair mechanisms of deteriorating white matter associated with aging [36]. Also consistent with this is an animal model of stroke that found elevated BDNF in white matter, suggesting that glial-mediated repair activity could mask an age-related decline of BDNF in white matter [37]. A repair-induced BDNF increase in white matter could confound an underlying age-related decline. However, given that we are unaware of other reports of an aging effect of BDNF in human white matter, and given the small sample in this study, a negative finding requires replication. Indeed, the results are based on a small sample and are preliminary. The lack of significantly reduced BDNF in schizophrenia PFC could also be due to the small sample, although a trend of reduced BDNF was observed (fig. 2). The finding of a slightly higher BDNF concentration in white matter was surprising, although again with a small sample size this must be retested. The study was designed to examine aging trends by including extreme age ranges, which could have reduced the robustness of group differences.

This study should also be viewed in light of the many limitations commonly associated with postmortem brain tissue, including differences in cause of death and some death-related processes not controlled by PMI, $\mathrm{pH}$ or RIN. We found no obvious relationship between causes of death and age or group differences in BDNF (data not shown). Another important limitation is using cross-sectional postmortem tissue data to study aging processes. Using cross-sectional data to infer longitudinal changes has significant limitations [38] and confirmation of our findings requires an alternative, longitudinal design [39], for example longitudinal animal studies. In this regard, longitudinal studies in animals support our BDNF aging trajectory observed in nonpsychiatric controls [6]. We did not measure receptor proteins in peripheral and brain tissue and test whether they follow the same age effect as the BDNF protein, which could be an interesting follow-up study given our initial finding on BDNF protein. Finally, imaging studies have frequently reported abnormal age-related gray and/or white matter declines in schizophrenia. It would be important to understand whether the age effect of BDNF plays a role in the different rates of gray and white matter decline with age between schizophrenia patients and healthy controls.

In conclusion, the answer to the question of whether BDNF can serve as a marker to delineate the neurodegeneration, or the lack thereof, across the adult lifespan of schizophrenia patients is complex but also revealing. BDNF in the PFC of older schizophrenia patients did not follow the normative linear decline. This blunting of the normative decline could be due to an early PFC BDNF decline or it could be a survivor cohort effect, both of which may be mechanisms indirectly supporting an abnormal brain aging process in some schizophrenia patients.

\section{Acknowledgments}

We thank Dr. Robert P. McMahon for statistical consultation on this paper. This research was supported by National Institutes of Health grants R01MH085646, P50MH10322, and R21MH079172 and by the Intramural Program of the National Institute on Aging. 


\section{References}

1 Kraepelin E: Dementia Praecox and Paraphrenia (Barclay RM, trans-ed), 1971 facsimile ed. Huntington, Krieger, 1919.

$\checkmark 2$ Knoll JL 4th, Garver DL, Ramberg JE, Kingsbury SJ, Croissant D, McDermott B: Heterogeneity of the psychoses: is there a neurodegenerative psychosis? Schizophr Bull 1998;24: 365-379.

3 Anderson JE, O’Donnell BF, McCarley RW, Shenton ME: Progressive changes in schizophrenia: do they exist and what do they mean? Restor Neurol Neurosci 1998;12:175-184.

4 McGlashan TH: The profiles of clinical deterioration in schizophrenia. J Psychiatr Res 1998;32:133-141.

5 Breier A, Schreiber JL, Dyer J, Pickar D: National Institute of Mental Health longitudinal study of chronic schizophrenia. Prognosis and predictors of outcome. Arch Gen Psychiatry 1991;48:239-246.

-6 Calabrese F, Guidotti G, Racagni G, Riva MA: Reduced neuroplasticity in aged rats: a role for the neurotrophin brain-derived neurotrophic factor. Neurobiol Aging 2013;34:2768-2776.

-7 Hariri AR, Weinberger DR: Imaging genomics. Br Med Bull 2003;65:259-270.

8 Nagappan G, Lu B: Activity-dependent modulation of the BDNF receptor TrkB: mechanisms and implications. Trends Neurosci 2005;28:464-471.

-9 Chapman TR, Barrientos RM, Ahrendsen JT, Hoover JM, Maier SF, Patterson SL: Aging and infection reduce expression of specific brain-derived neurotrophic factor mRNAs in hippocampus. Neurobiol Aging 2012;33:832. e1-e14.

10 Silhol M, Arancibia S, Maurice T, Tapia-Arancibia L: Spatial memory training modifies the expression of brain-derived neurotrophic factor tyrosine kinase receptors in young and aged rats. Neuroscience 2007;146:962-973.

- 11 Peng S, Wuu J, Mufson EJ, Fahnestock M: Precursor form of brain-derived neurotrophic factor and mature brain-derived neurotrophic factor are decreased in the pre-clinical stages of Alzheimer's disease. J Neurochem 2005;93:1412-1421.

12 Rao JS, Kim HW, Harry GJ, Rapoport SI, Reese EA: Increased neuroinflammatory and arachidonic acid cascade markers, and reduced synaptic proteins, in the postmortem frontal cortex from schizophrenia patients. Schizophr Res 2013;147:24-31.

13 Durany N, Michel T, Zochling R, Boissl KW, Cruz-Sanchez FF, Riederer P, Thome J: Brainderived neurotrophic factor and neurotrophin 3 in schizophrenic psychoses. Schizophr Res 2001;52:79-86.

14 Weickert CS, Hyde TM, Lipska BK, Herman MM, Weinberger DR, Kleinman JE: Reduced brain-derived neurotrophic factor in prefrontal cortex of patients with schizophrenia. Mol Psychiatry 2003;8:592-610.
15 Buckley PF, Mahadik S, Pillai A, Terry A Jr: Neurotrophins and schizophrenia. Schizophr Res 2007;94:1-11.

16 Chen da C, Wang J, Wang B, Yang SC, Zhang CX, Zheng YL, Li YL, Wang N, Yang KB, Xiu $\mathrm{MH}$, et al: Decreased levels of serum brainderived neurotrophic factor in drug-naive first-episode schizophrenia: relationship to clinical phenotypes. Psychopharmacology (Berl) 2009;207:375-380.

17 Jindal RD, Pillai AK, Mahadik SP, Eklund K, Montrose DM, Keshavan MS: Decreased BDNF in patients with antipsychotic naive first episode schizophrenia. Schizophr Res 2010;119:47-51.

18 Zalcman S, Endicott J: Diagnostic Evaluation after Death. New York, National Institute of Mental Health, 1983

19 Roberts SB, Hill CA, Dean B, Keks NA, Opeskin K, Copolov DL: Confirmation of the diagnosis of schizophrenia after death using DSM-IV: a Victorian experience. Aust NZJ Psychiatry 1998;32:73-76.

20 Li JZ, Vawter MP, Walsh DM, Tomita H, Evans SJ, Choudary PV, Lopez JF, Avelar A, Shokoohi V, Chung T, et al: Systematic changes in gene expression in postmortem human brains associated with tissue $\mathrm{pH}$ and terminal medical conditions. Hum Mol Genet 2004;13: 609-616.

21 Evans SJ, Choudary PV, Neal CR, Li JZ, Vawter MP, Tomita H, Lopez JF, Thompson RC, Meng F, Stead JD, et al: Dysregulation of the fibroblast growth factor system in major depression. Proc Natl Acad Sci U S A 2004;101: 15506-15511.

22 Tomita H, Vawter MP, Walsh DM, Evans SJ, Choudary PV, Li J, Overman KM, Atz ME, Myers RM, Jones EG, et al: Effect of agonal and postmortem factors on gene expression profile: quality control in microarray analyses of postmortem human brain. Biol Psychiatry 2004;55:346-352

23 Schroeder A, Mueller O, Stocker S, Salowsky R, Leiber M, Gassmann M, Lightfoot S, Menzel W, Granzow M, Ragg T: The RIN: an RNA integrity number for assigning integrity values to RNA measurements. BMC Mol Biol 2006;7:3.

24 Cohen J, Cohen P, West SG, Aiken LS: Applied Multiple Regression/Correlation Analysis for the Behavioral Sciences, ed 3. Mahwah, Lawrence Erlbaum Associates, 2003.

25 Kim HW, Cheon Y, Modi HR, Rapoport SI, Rao JS: Effects of chronic clozapine administration on markers of arachidonic acid cascade and synaptic integrity in rat brain. Psychopharmacology (Berl) 2012;222:663-674.

26 Luoni A, Berry A, Calabrese F, Capoccia S, Bellisario V, Gass P, Cirulli F, Riva MA: Delayed BDNF alterations in the prefrontal cortex of rats exposed to prenatal stress: preventive effect of lurasidone treatment during adolescence. Eur Neuropsychopharmacol 2014; 24:986-995.
27 Rizos E, Papathanasiou MA, Michalopoulou PG, Laskos E, Mazioti A, Kastania A, Vasilopoulou K, Nikolaidou P, Margaritis D, Papageorgiou $\mathrm{C}$, et al: A longitudinal study of alterations of hippocampal volumes and serum BDNF levels in association to atypical antipsychotics in a sample of first-episode patients with schizophrenia. PLoS One 2014; 9:e87997.

28 Buckley PF, Pillai A, Howell KR: Brain-derived neurotrophic factor: findings in schizophrenia. Curr Opin Psychiatry 2011;24:122127.

29 Chen CC, Huang TL: Effects of antipsychotics on the serum BDNF levels in schizophrenia. Psychiatry Res 2011;189:327-330.

30 Parks J, Svendsen D, Singer P, Foti ME: Morbidity and mortality in people with serious mental illness. Alexandria, NASMHPD, 2006.

31 Bushe CJ, Taylor M, Haukka J: Mortality in schizophrenia: a measurable clinical endpoint. J Psychopharmacol 2010;24(suppl 4): $17-25$.

32 Brown S: Excess mortality of schizophrenia. A meta-analysis. Br J Psychiatry 1997;171:502_508

33 Saha S, Chant D, McGrath J: A systematic review of mortality in schizophrenia: is the differential mortality gap worsening over time? Arch Gen Psychiatry 2007;64:1123-1131.

34 Douillard-Guilloux G, Guilloux JP, Lewis DA, Sibille E: Anticipated brain molecular aging in major depression. Am J Geriatr Psychiatry 2013;21:450-460.

35 Tsuang MT, Woolson RF: Excess mortality in schizophrenia and affective disorders. Do suicides and accidental deaths solely account for this excess? Arch Gen Psychiatry 1978;35: $1181-1185$.

36 Dalby RB, Elfving B, Poulsen PH, Foldager L, Frandsen J, Videbech P, Rosenberg R: Plasma brain-derived neurotrophic factor and prefrontal white matter integrity in late-onset depression and normal aging. Acta Psychiatr Scand 2013;128:387-396.

- 37 Sato Y, Chin Y, Kato T, Tanaka Y, Tozuka Y, Mase M, Ageyama N, Ono F, Terao K, Yoshikawa $\mathrm{Y}$, et al: White matter activated glial cells produce BDNF in a stroke model of monkeys. Neurosci Res 2009;65:71-78.

38 Kraemer HC, Yesavage JA, Taylor JL, Kupfer D: How can we learn about developmental processes from cross-sectional studies, or can we? Am J Psychiatry 2000;157: 163-171.

- 39 Thompson WK, Hallmayer J, O'Hara R: Alzheimer's disease neuroimaging. I. Design considerations for characterizing psychiatric trajectories across the lifespan: application to effects of APOE- $\varepsilon 4$ on cerebral cortical thickness in Alzheimer's disease. Am J Psychiatry 2011;168:894-903. 